\title{
Classical and quantum analysis of chaos in the discrete self-trapping equation
}

Cruzeiro-Hansson, Leonor; Feddersen, H.; Flesch, R.; Christiansen, Peter Leth; Salerno, Mario; Scott, Alwyn C.

Published in:

Physical Review B

Link to article, DOI:

10.1103/PhysRevB.42.522

Publication date:

1990

Document Version

Publisher's PDF, also known as Version of record

Link back to DTU Orbit

Citation (APA):

Cruzeiro-Hansson, L., Feddersen, H., Flesch, R., Christiansen, P. L., Salerno, M., \& Scott, A. C. (1990).

Classical and quantum analysis of chaos in the discrete self-trapping equation. Physical Review $B, 42(1), 522-$ 526. https://doi.org/10.1103/PhysRevB.42.522

\section{General rights}

Copyright and moral rights for the publications made accessible in the public portal are retained by the authors and/or other copyright owners and it is a condition of accessing publications that users recognise and abide by the legal requirements associated with these rights.

- Users may download and print one copy of any publication from the public portal for the purpose of private study or research.

- You may not further distribute the material or use it for any profit-making activity or commercial gain

- You may freely distribute the URL identifying the publication in the public portal 


\title{
Classical and quantum analysis of chaos in the discrete self-trapping equation
}

\author{
L. Cruzeiro-Hansson, ${ }^{*}$ H. Feddersen, R. Flesch, ${ }^{\dagger}$ P. L. Christiansen, \\ M. Salerno, ${ }^{\ddagger}$ and A. C. Scott \\ Laboratory of Applied Mathematical Physics, The Technical University of Denmark, DK-2800 Lyngby, Denmark
}

(Received 29 June 1989; revised manuscript received 16 February 1990)

\begin{abstract}
We study the discrete self-trapping model, for three degrees of freedom. The fraction of the energy shell of the phase space that is chaotic is evaluated directly from the classical motion and also from the exact energy levels of the corresponding quantum system. The correspondence between classical and quantum results is discussed.
\end{abstract}

\section{INTRODUCTION}

Several years ago the discrete self-trapping (DST) equation was introduced as a potentially interesting model of nonlinear dynamics. ${ }^{1}$ In simplest terms, the system comprises $f$ anharmonic oscillators (freedoms), which are coupled through linear dispersive interactions. More specifically, it takes the form

$$
\left(i \frac{d}{d t}-\omega_{0}\right) \bar{A}+\epsilon M \bar{A}+\gamma \operatorname{diag}\left(\left|A_{1}\right|^{2}, \ldots,\left|A_{f}\right|^{2}\right) \bar{A}=0,
$$

where $\bar{A}$ is the column vector

$$
\bar{A}=\operatorname{col}\left(A_{1}, A_{2}, \ldots, A_{f}\right)
$$

of complex components. With the dispersive parameter $\varepsilon$ and the anharmonic parameter $\gamma$ both equal to zero, Eq. (1.1) represents a system of $f$ noninteracting, harmonic oscillators each of frequency $\omega_{0}$. In the general case $(\epsilon \neq 0$ and $\gamma \neq 0)$, the dynamics of Eq. (1.1) exhibits the effects of both anharmonicity and dispersion. This interaction can lead to either self-trapping (i.e., local modes or solitons) or chaos or a mixture of self-trapping and chaos depending upon the parameters and initial conditions that are chosen. ${ }^{1}$

The original motivation for studying the DST equation arose from theoretical studies of vibrational energy selftrapping in protein ${ }^{2,3}$ and related experimental studies in hydrogen bonded, polypeptide crystals. ${ }^{4,5}$ In these applications the number of degrees of freedom $(f)$ is rather large: ca. 200 for a typical protein and much greater for an experimental polypeptide crystal. In order to gain theoretical perspective, initial attention was directed toward the DST with a few degrees of freedom, which is closely related to the study of anharmonic vibrations in small molecules [water, ammonia, methane, benzene, etc. (Refs. 6 and 7)]. During the course of these "chemical" investigations a quantum theory for the DST equation was developed $^{8,9}$ and has proven to be remarkably accurate and straightforward to apply. ${ }^{10}$

Our aim in this paper is twofold. First we augment previous studies of classical chaos exhibited by the DST equation; ${ }^{1,11-13}$ and, second, we relate these studies to the corresponding quantum analysis. Thus our work should be considered in the context of growing interest in "quantum chaology", ${ }^{14-20}$ but we believe that it is of particular interest for the following reasons. (i) The DS'T equation is a good model for anharmonic molecular vibrations ${ }^{4-9}$ and is therefore of considerable technical interest. (ii) As will be explained in the following, the quantum DST system can be analyzed without truncating infinite wave function expansions; ${ }^{8-10}$ thus the only errors in computing energy levels are numerical. (iii) The quantum DST system has an anharmonic parameter $-\gamma$ in Eq. (1.1) - which can be independently varied as one approaches the correspondence limit. These last two properties are shared by the spin system analyzed recently by Nakamura and Bishop. ${ }^{19}$ Motivated by the known mapping between an integrable nonlinear Schrödinger equation and an integrable spin system, ${ }^{21}$ Bishop has suggested that a mapping may exist between his nonintegrable spin system and the DST equation. ${ }^{22}$ We have studied this question in some detail and have been unable to find such a mapping. Even if one were to be found, however, we feel that property (i) of the DST system would be sufficient to justify the results presented here.

To appreciate the ease with which the DST system can be quantized, ${ }^{8,10}$ we note that it has two conserved quantities, the number

$$
N=\sum_{i=1}^{f}\left|A_{i}\right|^{2}
$$

and the energy

$$
H=\omega_{0} N-\frac{1}{2} \gamma \sum_{i=1}^{f}\left|A_{l}\right|^{4}-\epsilon \sum_{i \neq j}^{f} m_{i j} A_{i}^{*} A_{J} .
$$

Under quantization, the complex mode amplitudes ( $A_{i}^{*}$ and $A_{i}$ ) become boson creation and annihilation operators $\left(\hat{B}_{i}^{\dagger}\right.$ and $\left.\hat{B}_{i}\right)$.

With full symmetrization of the operator products Eq. (1.3) becomes the number operator

$$
\widehat{N}=\sum_{i=1}^{f}\left(\hat{B}_{i}^{\dagger} \hat{B}_{t}+\frac{1}{2}\right)
$$

and Eq. (1.4) becomes the energy operator 


$$
\begin{aligned}
\hat{\boldsymbol{H}}= & \left(\omega_{0}-\frac{1}{2} \gamma\right) \widehat{\boldsymbol{N}}-\frac{1}{2} \gamma \sum_{i=1}^{f} \widehat{\mathbf{B}}_{i}^{\dagger} \widehat{\boldsymbol{B}}_{i} \widehat{\boldsymbol{B}}_{i}^{\dagger} \widehat{\boldsymbol{B}}_{i} \\
& -\epsilon \sum_{i \neq j}^{f} m_{i j} \hat{\boldsymbol{B}}_{i}^{\dagger} \widehat{\boldsymbol{B}}_{j} .
\end{aligned}
$$

$\hat{H}$ and $\hat{N}$ operate upon wave functions of the form $\left|n_{1}\right\rangle\left|n_{2}\right\rangle \ldots\left|n_{f}\right\rangle$ which, for typographical convenience, we write as $\left[n_{1}, n_{2}, \ldots n_{f}\right]$.

Thus the quantized discrete self-trapping (QDST) equation describes an assembly of bosons. ${ }^{23}$ It has been discussed in some detail for nearest-neighbor interactions in the limit $\gamma \ll \epsilon$ and $f \rightarrow \infty .{ }^{24-26}$ This is the limit in which the classical DST reduces to the nonlinear Schrödinger equation. ${ }^{27}$

Stationary states of the QDST equation must be eigenfunctions of both $\hat{N}$ and $\hat{H}$. As in Refs. 7 and 8 , the wave function, $\left|\psi_{n}\right\rangle$, is chosen to be a linear combination of all possible states of $n$ particles, leading to a matrix equation for the column vector consisting of the coefficients in this expansion.

It is now well established that classical solution trajectories of Eq. (1.1) for three or more degrees of freedom $(f \geq 3)$ can be either chaotic or quasiperiodic for appropriate values of the dispersive and anharmonic parameters, $\epsilon$ and $\gamma \cdot{ }^{1,6,11-13}$ Here we study the system for $f=3$ with the dispersion matrix

$$
\boldsymbol{\epsilon} \boldsymbol{M}=\left(\begin{array}{lll}
0 & 1 & 1 \\
1 & 0 & 1 \\
1 & 1 & 0
\end{array}\right)
$$

from the perspectives of both classical and quantum dynamics.

Results of the classical studies are presented in the following section. Here we estimate the fraction of phase space occupied by chaotic trajectories, which we call $\rho_{c}$. Quantum results are presented in Sec. III. We use tools that have been developed by Berry to estimate again the chaotic fraction of phase space, called $\rho_{q} \cdot{ }^{20}$ If these tools are valid we expect $\rho_{q} \rightarrow \rho_{c}$ as $n \rightarrow \infty$, the correspondence limit. The degree to which we are able to confirm such a correspondence is discussed in the concluding section.

\section{CLASSICAL ANALYSIS}

The DST equation for one degree of freedom reduces to a nonlinear oscillator and the solution is sinusoidal. For two degrees of freedom, we have two conserved quantities and again the system is integrable. Indeed, it has been shown that it can be reduced to the pendulum equation. ${ }^{28,29}$ Here we shall confine ourselves to the three degrees of freedom DST which are not integrable. Indeed, chaotic trajectories have been found. ${ }^{1,11-13}$ The latter studies were done fixing a specific initial condition and varying the nonlinearity parameter $\gamma$. In a particular case, by varying $\gamma$, regular trajectories were encountered in a narrow window amidst chaotic ones. ${ }^{13}$ However, because these results are valid for one particular initial condition, one cannot claim that such a regular window is a generic feature for this narrow range of values. To do so, one must use a global indicator which probes the entire energy shell. Such an indicator is $\rho_{c}$, which is defined as the fraction of the energy shell $E$, which is chaotic. ${ }^{30}$

In the case of DST, the phase space must be restricted to the constant $N$ surface as well as the energy shell $E$ when the classical results are compared with quantum results.

Two methods of evaluating $\rho_{c}$ have been investigated. Meyer relates an integral over the energy surface to an integral over the Poincaré section. ${ }^{30}$ Implicit in his derivation is the assumption that all trajectories on the energy surface intersect the Poincare section. We have not found any Poincaré section for which this assumption is valid in our system and suspect that in general it is not true. ${ }^{31}$ For this reason we have adopted a second method to evaluate $\rho_{c}$.

The second method, on which all of our classical results are based, is a Monte Carlo evaluation ${ }^{32}$ of $\rho_{c}$. We choose a number of random initial conditions which are uniformly distributed on the energy surface. These initial conditions must also have the same value of $N$. The fraction of these initial conditions which leads to chaotic trajectories is then our estimate of $\rho_{c}$.

The obvious test of whether or not a trajectory is chaotic is to calculate the maximal Lyapunov exponent, ${ }^{30,32}$ but because of excessive computational costs we chose instead to determine whether or not the trajectory is chaotic by examination of a Poincare section.

In Fig. 1 we show a Poincare section representative of those which are encountered when the determination of chaotic versus regular is made. The dots in the figure all belong to a trajectory, which is labeled chaotic. The crosses, which form a nearly closed curve, belong to a quasiperiodic, i.e., regular orbit. For two energies $(\gamma E=-0.1$ and $\gamma E=-0.6)$ we examined both the maximal exponents and the Poincare sections. Good agreement on the value of $\rho_{c}$ was obtained.

In roughly $95 \%$ of the sections studied, the determination of whether or not the trajectory is chaotic is quite

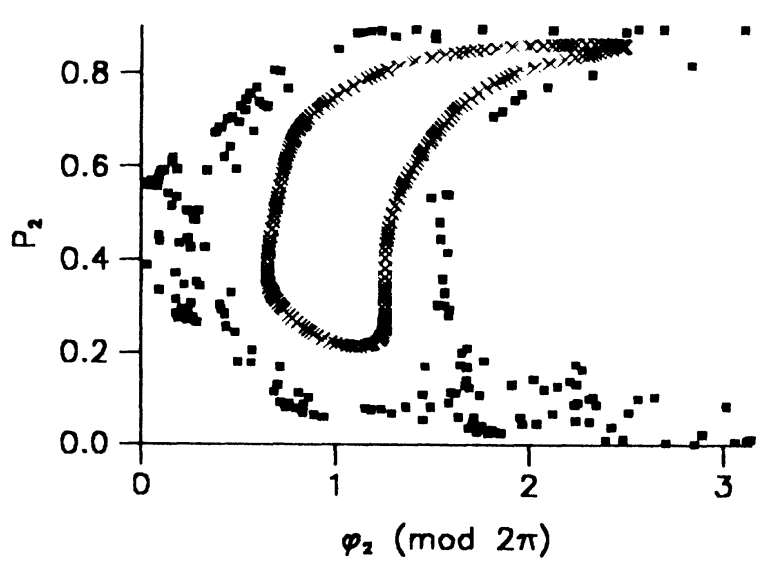

FIG. 1. Poincaré section of regular $(X)$ and chaotic $(\square)$ trajectories. $\left(P_{1}=0.1, \gamma N=3, \gamma E=-2.4\right.$. $)$ 
unambiguous as in Fig. 1. Any errors introduced in this way are small in comparison with the statistical error associated with the Monte Carlo method.

Instead of working with the original variables, $A_{i}$ and $A_{i}^{*}$ (which are canonical variables with respect to the Hamiltonian $-i H$ ), we have made the following transformation to canonical variables:

$$
\begin{aligned}
& A_{1}=\sqrt{P_{1}} e^{-i\left(\phi_{1}+\phi_{3}\right)}, \quad 0<P_{1}<N, \\
& A_{2}=\sqrt{P_{2}} e^{-i\left(\phi_{2}+\phi_{3}\right)}, \quad 0<P_{2}<N, \\
& A_{3}=\sqrt{P_{3}-P_{1}-P_{2}} e^{-i \phi_{3}}, \quad 0<P_{1}+P_{2}<N,
\end{aligned}
$$

leading to the Hamiltonian

$$
\begin{aligned}
H(\phi, \mathbf{P})=-\frac{1}{2} \gamma[ & \left.P_{1}^{2}+P_{2}^{2}+\left(N-P_{1}-P_{2}\right)^{2}\right] \\
-2 \epsilon[ & \sqrt{P_{1} P_{2}} \cos \left(\phi_{1}-\phi_{2}\right) \\
& +\sqrt{P_{1}\left(N-P_{1}-P_{2}\right)} \cos \phi_{1} \\
& \left.+\sqrt{P_{2}\left(N-P_{1}-P_{2}\right)} \cos \phi_{2}\right],
\end{aligned}
$$

where $P_{i}$ is the momentum conjugate to $\phi_{i}, i=1,2,3$. In this description the momentum $P_{3}$ is in fact the conserved quantity $N$. Hence $\phi_{3}$ is an ignorable coordinate. Thus the equations of motion become

$$
\begin{aligned}
& \dot{\phi}_{1}=\frac{\partial H}{\partial P_{1}}, \quad \dot{\phi}_{2}=\frac{\partial H}{\partial P_{2}}, \\
& \dot{P}_{1}=-\frac{\partial H}{\partial \phi_{1}}, \quad \dot{P}_{2}=-\frac{\partial H}{\partial \phi_{2}} .
\end{aligned}
$$

(The time evolution of $\phi_{3}$, which is determined by the equation $\dot{\phi}_{3}=\partial H / \partial N$ may be chaotic.) Note that Eq. (1.1) is invariant under the transformations $A_{j} \rightarrow \alpha A_{j}, \gamma \rightarrow \alpha^{-2} \gamma, N \rightarrow \alpha^{2} N$, and $H \rightarrow \alpha^{2} H$. Since $\gamma N$ and $\gamma H$ are unchanged under these transformations they are used to specify the number and the energy, respectively. ${ }^{12}$

For the purpose of computing $\rho_{c}$, the preceding transformation effectively reduces the number of degrees of freedom from three to two. The initial conditions are randomly distributed over the energy surface $H\left(P_{1}, P_{2}, \phi_{1}, \phi_{2}\right)=E$. Thus, $\phi_{1}$ becomes a function of $P_{1}, P_{2}$, and $\phi_{2}$.

The random initial conditions for $P_{1}, P_{2}$, and $\phi_{2}$ are weighted by the projection of the surface element $\left(P_{1}, P_{2}, \phi_{1}, \phi_{2}\right) \rightarrow\left(P_{1}, P_{2}, \phi_{2}\right)$.

Thus $\rho_{c}$ can be calculated from the trajectories determined by (2.3) for any fixed values of $\gamma E$ and $\gamma N$. (The value $\gamma N=3$ is chosen throughout.)

Figure 2 shows our results for $\rho_{c}$, based on 50-100 trajectories (vertical error bars), as well as the corresponding quantity, $\rho_{q}$ (horizontal error bars), in the quantummechanical case considered in Sec. III. The error bars in $\rho_{c}$ are determined as the $95 \%$ confidence interval of our estimate of $\rho_{c}$. In the limits of high and low $\gamma E$ the DST equation is integrable. As a consequence, $\rho_{c} \rightarrow 0$ in these limits is seen in the figure. Local minima are found at $\gamma E \sim-4.2$ and -3.8 , which may be interpreted as windows of less chaotic behavior. However, the window for

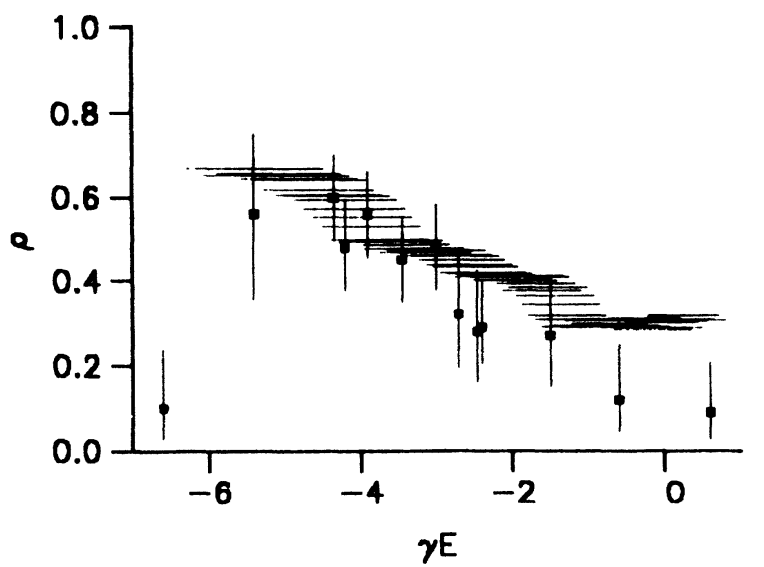

FIG. 2. $\rho_{c}(\boldsymbol{\square})$ and $\rho_{q}(-)$ as functions of energy $\gamma E$ for fixed $\gamma N=3.00$. Error bounds indicated by vertical and horizontal bars. $n=310$ in the QDST giving a total of 8164 energy levels.

$\gamma E=-10.99$ and $\gamma N=4.5$ reported in Ref. 13 was found to give a value $\rho_{c} \sim 0.73-0.98$. Thus, although the particular initial condition considered was regular for the latter values of $\gamma N$ and $\gamma E$, about $90 \%$ of the energy shell is actually occupied by chaotic trajectories.

\section{QUANTUM ANALYSIS}

In this section we study the quantum counterpart of the DST equation with three freedoms. Studies of energy spectra of quantum systems have shown that the density of spacings, $S$, between uncorrelated, adjacent energy levels, $p(S)$, can be used as an indicator of chaos or regularity in the corresponding classical systems. ${ }^{14-18,32-38}$.

When the classical phase space is partitioned into regular regions and only one chaotic region the following interpolation formula has been proposed: ${ }^{33}$

$$
\begin{aligned}
p_{I}(S)= & \exp \left[-\left(1-\rho_{q}\right) S-\frac{\pi}{4} \rho_{q}^{2} S^{2}\right] \\
\times & {\left[\left(1-\rho_{q}\right)^{2} \exp \left[\frac{\pi}{4} \rho_{q}^{2} S^{2}\right] \operatorname{erfc}\left[\frac{\sqrt{\pi}}{2} \rho_{q} S\right]\right.} \\
& \left.+2\left(1-\rho_{q}\right) \rho_{q}+\frac{\pi}{2} \rho_{q}^{3} S\right] .
\end{aligned}
$$

Here $\rho_{q}$ is the fraction of the energy shell that is chaotic.

Equation (3.1) interpolates between the Poisson distribution (for the quantum counterpart of the regular classical system ${ }^{34}$ ) and the Wigner distribution [corresponding to the chaotic classical system (Ref. 14)].

Equation (3.1) shows how the fraction of the energy shell that is chaotic in the classical system can be determined from the energy levels of the corresponding quantum system. Its use implies the assumption that all chaotic regions are connected. In the case of the DST equation with three degrees of freedom, which effectively is a system with two degrees of freedom (see Sec. II), this must be assumed in order to make a unique fitting to the 
available data. Hence Eq. (3.1) is used as an approximation. $^{18}$

Above we have specified that the spacings distribution should be done with uncorrelated energy levels. By uncorrelated we mean levels that evolve independently when parameters in the Hamiltonian (such as nonlinearity $\gamma$ ) are varied. In the case of the DST model this means, for instance, that states corresponding to different $n$ values must not be mixed. Also, and generally for all Hamiltonians that possess symmetry properties, the energies must be separated according to the symmetry groups in order to avoid degenerate levels.

The results shown in this article were obtained from energy sequences corresponding to the symmetric states, the number of which is $1+\operatorname{int}[n(n+6) / 12] .^{10} \mathrm{~A}$ final remark is that the spacings distribution should be determined from an energy sequence that possesses a large number of energy levels in a short energy range. This usually implies large quantum numbers and correspondingly large matrices to diagonalize, which is impossible in practice. Energy sequences with lower density of levels can also be used, provided they have been scaled previously into other sequences that have constant density. In this way the secular variation in the density is eliminated in order that the short range (expressed by the spacings distribution) be brought to light. ${ }^{38}$

Figure 2 shows the variation of $\rho_{q}$ with $\gamma E$ (for $\gamma n=3$ ). Each $\rho_{q}$ value is calculated from a sequence with 2000 levels, the energy $E$ being the average energy and the error being the corresponding standard deviation. Superimposed on them are the values of $\rho_{c}$. Our numerical calculations show that at least 1500 levels are needed for reliable statistics.

The quantum results confirm the trend of the classical ones. However, the correspondence is not as good as that found by previous authors. ${ }^{15,18,36,38}$ One reason may be that with the value of $n$ used, the density of levels is not large enouch (a fact that is expressed by the horizontal error bars). A clear divergence from the classical result is observed in the low $\gamma E$ limit. This is due to the particularly low density of quantum levels in this region (with the correspondingly larger horizontal error bars). Our conclusion is that in the low $\gamma E$ region, for the values of $n$ used, the $\rho_{q}$ determined cannot be trusted. In the other regions, on the other hand, the quantum calculations correspond to an averaging over a range of classical energy shells. This leads to a loss of the more detailed structure indicated by the classical calculation.

\section{CONCLUSIONS}

The fundamental aim of this paper is to study quantum and classical determinations of the chaotic fraction of the energy shell in phase space for the discrete self-trapping equation with three degrees of freedom. The classically computed fraction is called $\rho_{c}$, and the fraction determined quantum mechanically using the procedure outlined by Berry and co-workers ${ }^{20,33,34}$ is called $\rho_{q}$.

Our results are presented in Fig. 2. In the intermediate range of energy, where $\rho_{c} \cong \rho_{q} \cong \frac{1}{2}$, the agreement seems to be within the error bars. At the extreme values of energy, however, the agreement is not good. This may be because the system is not generic ${ }^{39}$ at the extreme values, or because the number of levels chosen (8164) is not sufficient.

\section{ACKNOWLEDGMENTS}

The financial support of the Danish Research Council for Scientific and Industrial Research, NATO Scientific Affairs Division through Grant No. RG-8510396, the Gulbenkian Foundation, Lisbon, Portugal, Thomas B. Thriges Fond, Denmark, is acknowledged by the authors L.C.-H., R.F., and M.S.
*Present address: Department of Crystallography, Birkbeck College, University of London, Malet Street, London WC1E 7HX, United Kingdom.

†Present address: Heriot-Watt University, Department of Mathematics, Riccarton, Edinburgh EH14 4AS, Scotland.

$\ddagger$ Permanent address: Dipartimento di Fisica Teoretica, Università di Salerno, I-84100 Salerno, Italy.

${ }^{1}$ J. C. Eilbeck, P. S. Lomdahl, and A. C. Scott, Physica D 16, 318 (1985).

${ }^{2}$ A. S. Davydov, Biology and Quantum Mechanics (Pergamon, Oxford, 1981).

${ }^{3}$ A. S. Davydov, Sov. Phys. Usp. 25, 899 (1982) [Usp. Fiz. Nauk 138, 603 (1982)].

${ }^{4}$ G. Careri, U. Buontempo, F. Galluzzi, A. C. Scott, E. Gratton, and E. Shyamsunder, Phys. Rev. B 30, 4689 (1984).

${ }^{5}$ J. C. Eilbeck, P. S. Lomdahl, and A. C. Scott, Phys. Rev. B 30, 4703 (1984).

${ }^{6}$ A. C. Scott, P. S. Lomdahl, and J. C. Eilbeck, Chem. Phys. Lett. 113, 29 (1985).

${ }^{7}$ A. C. Scott and J. C. Eilbeck, Chem. Phys. Lett. 132, 23 (1986).

${ }^{8}$ A. C. Scott and J. C. Eilbeck, Phys. Lett. A 119, 60 (1986).

${ }^{9}$ A. C. Scott, L. Bernstein, and J. C. Eilbeck, J. Biol. Phys. 17, 1
(1989); L. Bernstein, J. C. Eilbeck, and A. C. Scott, Nonlinearity (to be published).

${ }^{10}$ S. de Filippo, M. Fusco Girard, and M. Salerno, Nonlinearity (to be published).

${ }^{11}$ J. H. Jensen, P. L. Christiansen, J. N. Elgin, J. D. Gibbon, and O. Skovgaard, Phys. Lett. 110A, 429 (1985).

${ }^{12}$ S. de Filippo, M. Fusco Girard, and M. Salerno, Physica D 26, 411 (1987).

${ }^{13}$ S. de Filippo, M. Fusco Girard, and M. Salerno, Physica D 29, 421 (1988).

${ }^{14}$ O. Bohigas, M. J. Giannoni, and C. Schmit, Phys. Rev. Lett. 54, 1 (1984).

${ }^{15}$ E. Haller, H. Köppel, and L. S. Cederbaum, Phys. Rev. Lett. 52, 1665 (1984).

${ }^{16}$ G. Casati, B. V. Chirikov, and I. Guarneri, Phys. Rev. Lett. 54, 1350 (1985).

${ }^{17}$ Chaotic Behavior in Quantum Systems, edited by G. Casati (Plenum, New York, 1985).

${ }^{18}$ T. Zimmerman, H. D. Meyer, H. Köppel, and L. S. Cederbaum, Phys. Rev. A 33, 4334 (1986).

${ }^{19}$ K. Nakamura and A. R. Bishop, Phys. Rev. B 33, 1963 (1986).

${ }^{20}$ M. V. Berry, Proc. R. Soc. (London) A 413, 183 (1987). 
${ }^{21}$ Y. Ishimori, J. Phys. Soc. Jpn. 51, 3417 (1982).

${ }^{22}$ A. R. Bishop (private communication).

23P. A. M. Dirac, The Principles of Quantum Mechanics, 4th ed. (Clarendon, Oxford, 1958), pp. 227-232.

${ }^{24}$ E. K. Sklyanin and L. D. Faddeev, Sov. Phys. Dokl. 23, 902 (1978) [Dokl. Adad. Nauk SSSR 243, 1430 (1978)].

${ }^{25}$ H. B. Thacker and D. Wilkinson, Phys. Rev. D 11, 3660 (1979).

${ }^{26}$ M. Wadati in Dynamical Problems in Soliton Systems, edited by S. Takeno (Springer, Berlin, 1985), pp. 68-80.

${ }^{27}$ V. E. Zakharov and A. B. Shabat, Sov. Phys.-JETP 34, 62 (1972) [Zh. Eksp. Teor. Fiz. 61, 118 (1971)].

${ }^{28}$ L. Cruzeiro-Hansson, P. L. Christiansen, and J. N. Elgin, Phys. Rev. B 37, 7896 (1988).

${ }^{29}$ A. C. Scott and P. L. Christiansen, Phys. Scr. (to be published).

${ }^{30}$ H.-D. Meyer, J. Chem. Phys. 84, 3147 (1986).
${ }^{31}$ H. K. Feddersen, R. Flesch, M. Salerno, and P. L. Christiansen, J. Chem. Phys. 92, 2117 (1990).

${ }^{32}$ T. H. Seligman and J. J. M. Verbaarschot, J. Phys. A 18, 2227 (1985).

${ }^{33}$ M. V. Berry and M. Robnik, J. Phys. A 17, 2413 (1984).

${ }^{34}$ M. V. Berry and M. Tabor, Proc. R. Soc. (London) A 356, 375 (1977).

${ }^{35}$ P. Pechukas, Phys. Rev. Lett. 51, 943 (1983).

${ }^{36}$ T. H. Seligman, J. J. M. Verbaarschot, and M. R. Zirnbauer, Phys. Lett. 53, 215 (1984).

${ }^{37}$ D. Wintgen and H. Friedrich, Phys. Rev. A 35, 1464 (1987).

${ }^{38}$ E. Haller, H. Köppel, and L. S. Cederbaum, Chem. Phys. Lett. 101, 215 (1983).

${ }^{39}$ M. V. Berry, in Chaotic Behavior of Deterministic Systems, edited by G. Iooss, R. H. G. Helleman, and R. Stora (NorthHolland, Amsterdam, 1983), p. 171. 